\title{
ANÁLISIS NO LINEAL DEL AISLADOR DE TRIPLE PÉNDULO DE FRICCIÓN MEDIANTE EL MODELO TRI- LINEAL
}

\section{NONLINEAR DYNAMIC ANALYSIS OF TRIPLE FRICTIONAL PENDULUM BEARING THROUGH TRI- LINEAR MODEL}

\author{
Pablo Leiva, Roberto Aguiar \\ Departamento de Ciencias de la Tierra y la Construcción \\ Universidad de Fuerzas Armadas ESPE \\ Av. Gral. Rumiñahui s/n. Valle de los Chillos, Ecuador \\ psleiva@espe.edu.ec \\ rraguiar@espe.edu.ec
}

\begin{abstract}
RESUMEN
En la Universidad de Fuerzas Armadas ESPE, desde el 2009, el doctor Roberto Aguiar viene desarrollando el sistema de computación CEINCI-LAB para el análisis estático y dinámico de estructuras. Hasta el momento se ha utilizado el modelo bilineal para determinar el desempeño del aislador de Triple Péndulo de Fricción. La presente investigación plantea comparar el modelo bilineal con el trilineal propuesto por McVitty y Constantinou (2015). Para ello se va a desarrollar un nuevo aplicativo en el software Matlab del modelo tri-lineal para un análisis respuesta en el tiempo con un grado de libertad e incorporar al CEINCI-LAB.
\end{abstract}

Palabras Claves: Aislador de Triple Péndulo de Fricción. Modelo bilineal. Modelo tri-lineal. Sistema de computación CEINCI-LAB.

\begin{abstract}
At Universidad de las Fuerzas Armadas ESPE, Roberto Aguiar has been developing the CEINCI-LAB computing system for static and dynamic structural analysis since 2009. Until now the bilinear model has been used to determine the performance of Triple Frictional Pendulum bearing. This paper will compare the bilinear model with the tri-linear model proposed by McVitty y Constantinou (2015). For this comparison, a new application developed with Matlab software of the trilinear model will be used for the response time analysis with one degree of freedom, and will incorporate CEINCI-LAB.
\end{abstract}

Keywords: Triple Frictional Pendulum bearing. Bilinear model. Tri-linear model. System of CEINCI-LAB computer. 


\section{INTRODUCCIÓN}

El Ecuador se encuentra ubicado dentro del cinturón de fuego del Pacífico, que es la zona de más alto riesgo sísmico y volcánico en el mundo, como se pudo evidenciar el pasado 16 de abril de 2016, con un sismo de magnitud 7.8. Estos eventos sísmicos y el avance tecnológico en la construcción de edificaciones con aislación sísmica han motivado al Ecuador a no quedarse atrás. Actualmente en el país existe una gran cantidad de estructuras con aislación sísmica, se detalla a continuación algunos de ellos:

Puente "Los Caras": Este puente se encuentra ubicado al norte de la provincia de Manabí, une a San Vicente con Bahía de Caráquez, con una longitud de 1980 metros. Dispone de 160 aisladores de Triple Péndulo de Fricción, FPT (Triple Frictional Pendulum) y los ubicaron entre las bases del puente que se encuentran sobre el agua y la vía por donde circulan los vehículos.

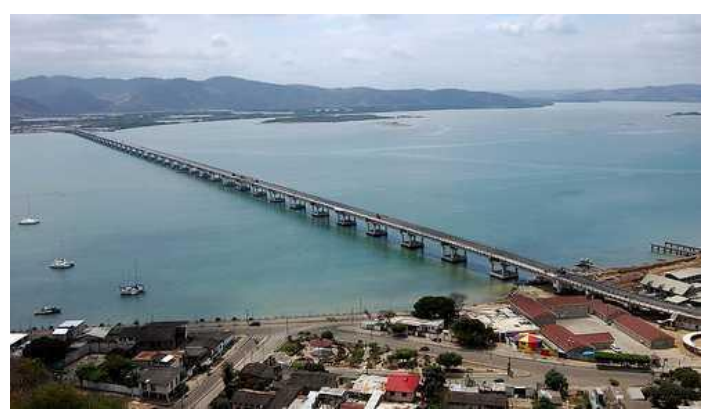

Figura 1 Puente "Los Caras" en Bahía de Caráquez Fuente: http://www.tctelevision.com/

Puentes de Esmeraldas: La construcción de los puentes sobre el Estuario del río Esmeraldas permiten el acceso al Puerto Marítimo, al Aeropuerto de Tachina y el ingreso a la ciudad de Esmeraldas, en la Figura 2 se observa la ubicación de cada uno, tres de los puentes se encuentran construidos con aisladores de base FPT.

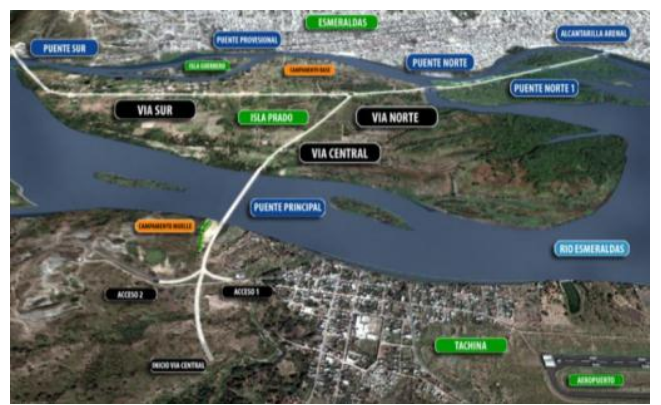

Figura 2 Ubicación de los Puentes.

Fuente: Vergara Navarrete (2015)

Los Puentes construidos con aisladores FPT son: el Puente Sur, el Puente Norte y el Puente Norte 1. El Puente Principal de longitud de $434 \mathrm{~m}$ se encuentra construido con apoyos de neopreno. 
- Puente Sur: se encuentra ubicado en el sur de la Isla Prado y en uno de los brazos que complementa el Estuario del río Esmeraldas, tiene una longitud de 160 metros, un ancho de 18,90 metros y 12 aisladores de Triple Péndulo de fricción, tres por cada estribo y tres por pila, con una capacidad de desplazamiento de 23 pulgadas $(58,42 \mathrm{~cm})+/-0,3$ pulgadas $(0,762 \mathrm{~cm})$.

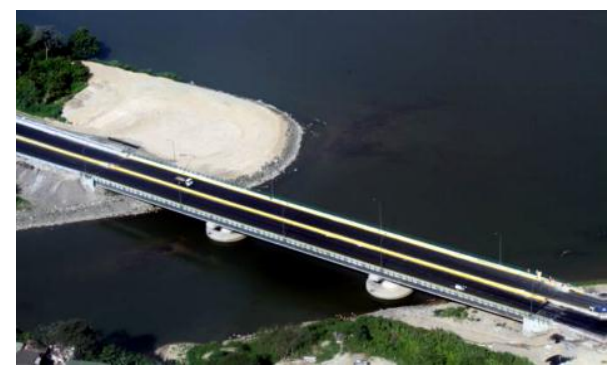

Figura 3 Puente Sur

Fuente: Guaygua (2015)

- Puente Norte: se encuentra ubicado al norte de la Isla Prado y en uno de los brazos que complementa el Estuario del río Esmeraldas, tiene una longitud de 120 metros, un ancho de 18,90 metros y 9 aisladores de Triple Péndulo de Fricción, con las mismas características del Puente Sur.

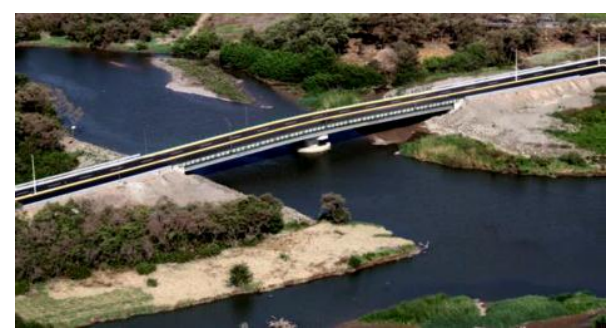

Figura 4 Puente Norte

Fuente: Guaygua (2015)

- Puente Norte 1: se encuentra ubicado norte de la Isla Prado cerca del sector de la marina, tiene una longitud de 108 metros, un ancho de 18,90 metros y 9 aisladores de Triple Péndulo de fricción.
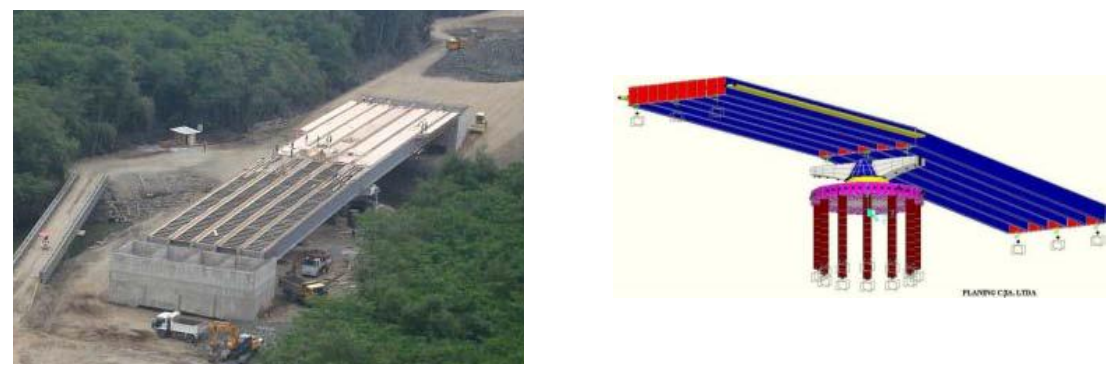

Figura 5 Puente Norte 1

Fuente: Vergara Navarrete (2015) 
Aeropuerto Internacional Mariscal Sucre: es el nuevo aeropuerto de Quito, se encuentra ubicado en la parroquia de Tababela. Con las siguientes características operacionales, Quiport (2013): una altitud de 2400 metros sobre el nivel del mar, una pista de 4100 metros de largo, la Torre de Control es de 41 metros de altura, el Parqueo de aeronaves tiene 45 puestos, el área para el terminal de pasajeros es 38000 metros cuadrados y el terminal de carga es 14000 metros cuadrados. En el Puente Peatonal están colocados 16 aisladores sísmicos de dos tipos debido a las solicitaciones en el diseño.

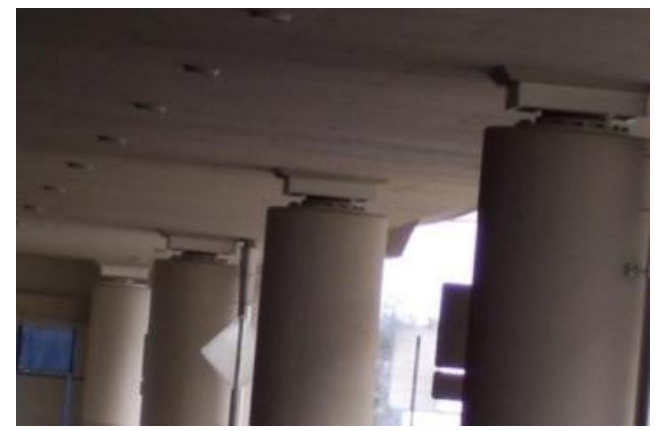

Figura 6 Aisladores Sísmico en el puente peatonal del Aeropuerto de Quito. Fuente: Quiport

Edificio Sede de UNASUR: se encuentra ubicado en la Mitad del Mundo, con un área de $20.000 \mathrm{~m} 2$ de construcción. Es una estructura que debe responder en el rango elástico, núcleo con muros acoplados de hormigón armado sobre cimentación continua, dos volados con celosías de acero (38 y 50 metros) y pisos aislados en 3 dimensiones al interior de los volados, en la Figura 7 se presenta el Edificio Sede de UNASUR, las celosías de aceros y el aislador 3D.

Criterio de diseño del sistema de aislación en los volados:

- Reducir los niveles de aceleración horizontal y vertical en los pisos de oficinas para evitar el daño a componentes no-estructurales y equipos y la conmoción de los ocupantes en caso de un terremoto.

- Mitigar las vibraciones generadas por actividades humanas en los pisos de oficinas.

- Reducir la demanda sísmica en los elementos de la estructura en celosía que soporta a los volados.

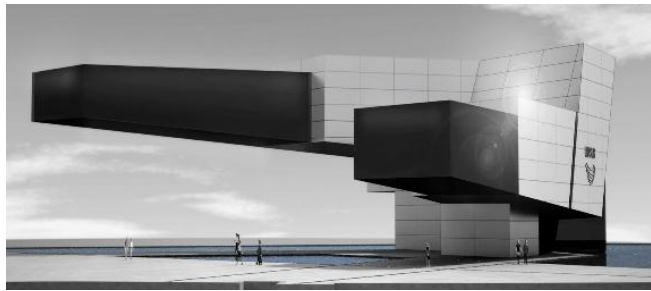

a)

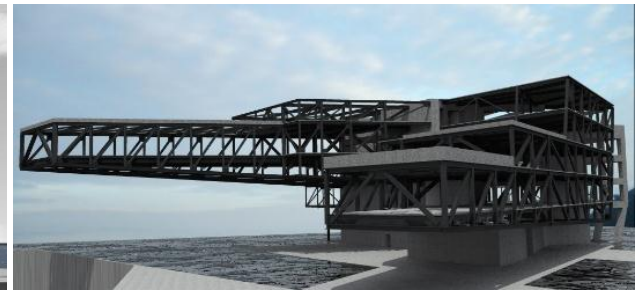

b) 

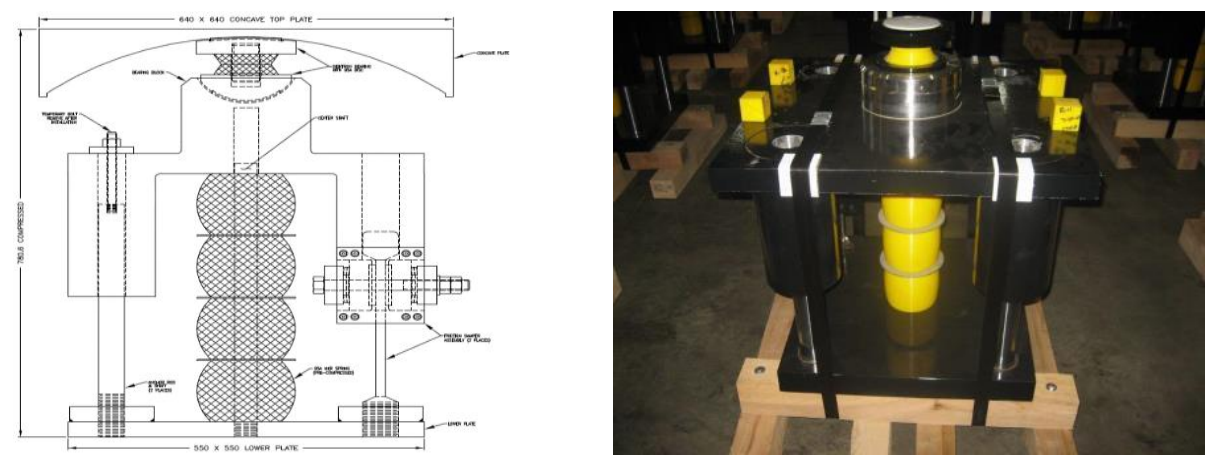

c)

Figura 7 a) Perspectiva del Edificio Sede de UNASUR; b) Volados y celosías de aceros; c) Aislador 3D Fuente: Suárez (2013)

Centro de Investigación y Posgrado de la Universidad de las Fuerzas Armadas - ESPE: es el nuevo Centro de Investigaciones y Posgrado de la UFAESPE, para la construcción de este proyecto se han requerido de ocho bloques estructurales que contarán con 81 aisladores de Triple Péndulo de Fricción y se implantará en un área de 23 mil 338 metros cuadrados. En la Figura 8 se observa una perspectiva en 3 dimensiones del proyecto, la implantación y la etapa constructiva de los cinco bloques estructurales con aislación sísmica.

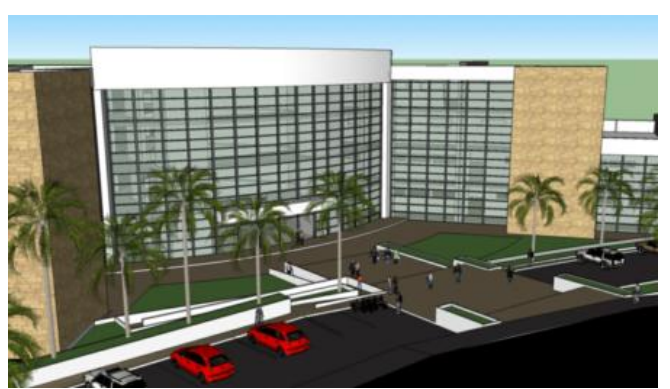

a)

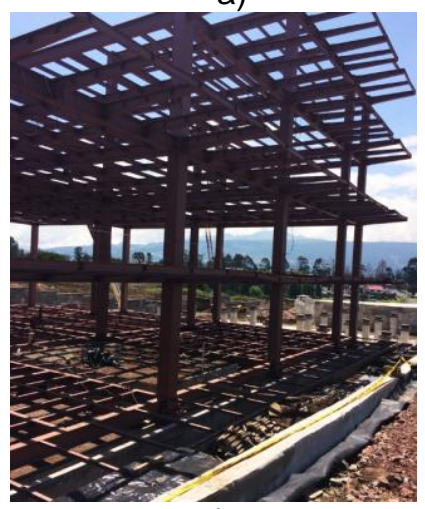

c)

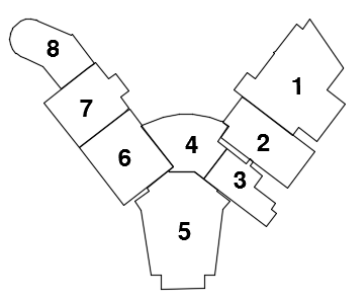

b)

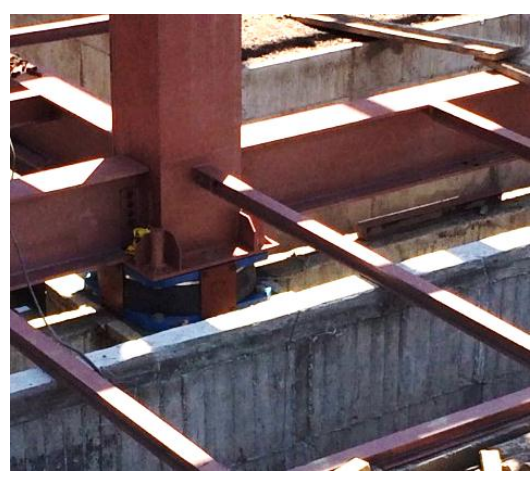

d) 


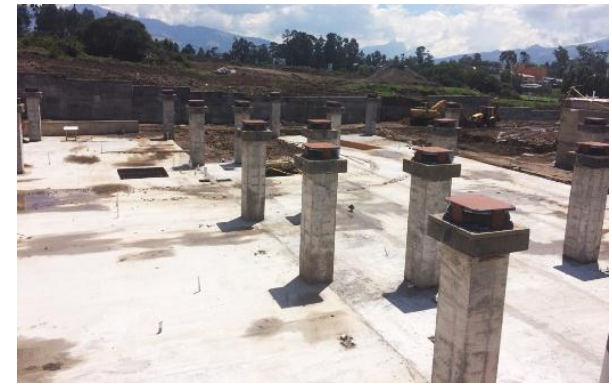

e)

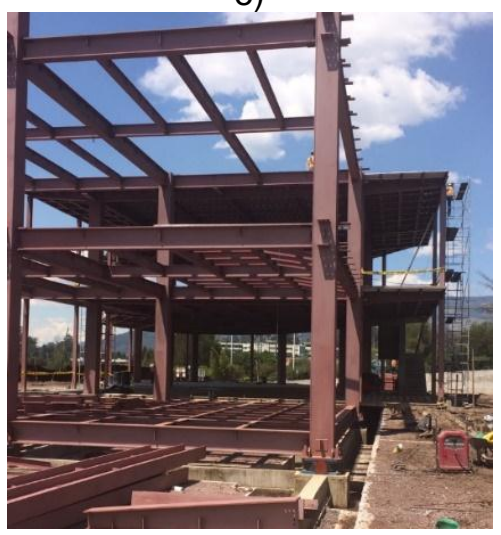

g)

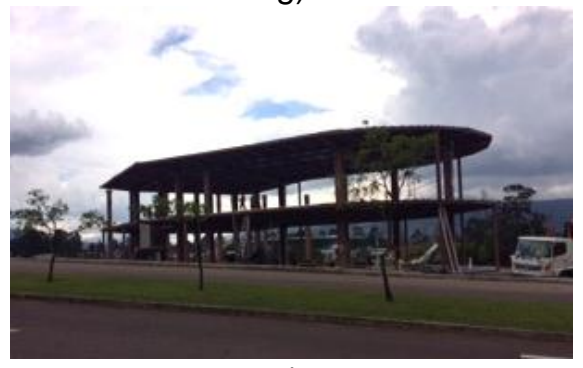

i)

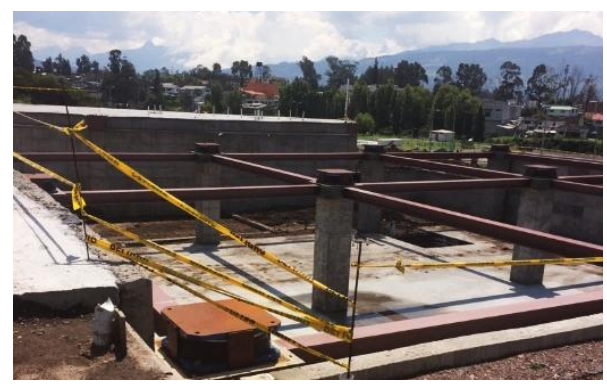

f)

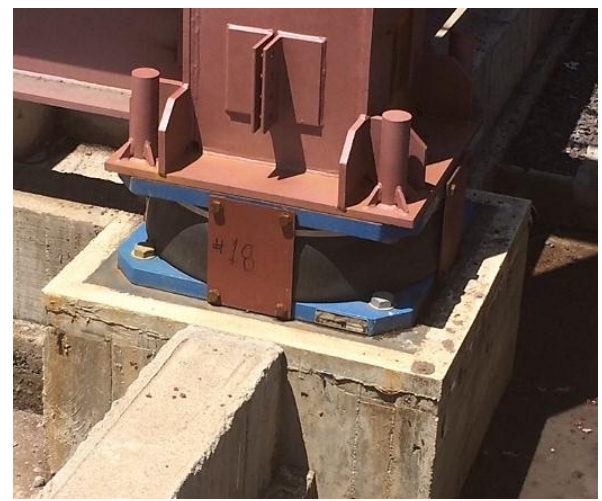

h)

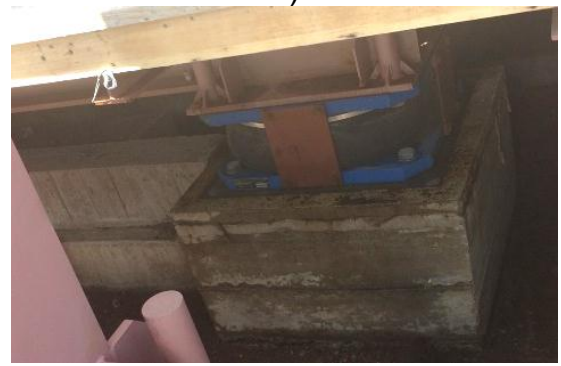

j)

Figura 8 Centro de Investigaciones y Posgrado UFA-ESPE; a) Perspectiva Noreste; b) Ubicación de los bloques estructurales; Fotografías de la etapa constructiva hasta octubre del 2016 c) Bloque 1; d) Aislador del Bloque 1; e) Colocación de aisladores en el Bloque 2; f) Colocación de aisladores en el Bloque 6; g) Bloque 7; h) Aislador Bloque 7; i) Bloque 8; j) Aislador Bloque 8.

Sky Building: es un nuevo proyecto inmobiliario ubicado en la salida norte del aeropuerto internacional José Joaquín de Olmedo, en Guayaquil. Esta edificación diseñada con aisladores sísmicos es de 10 pisos, con 216 oficinas y 3 locales comerciales. Los aisladores se colocaron sobre las columnas del subsuelo y el bloque de ascensor y gradas está separado del bloque principal estructural para colocar los aisladores sobre la cimentación, observar Figura 9. 


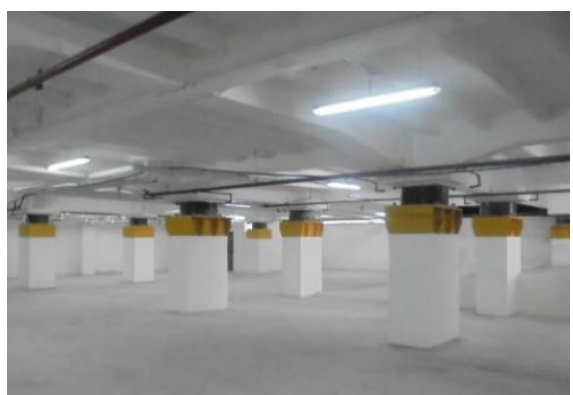

a)

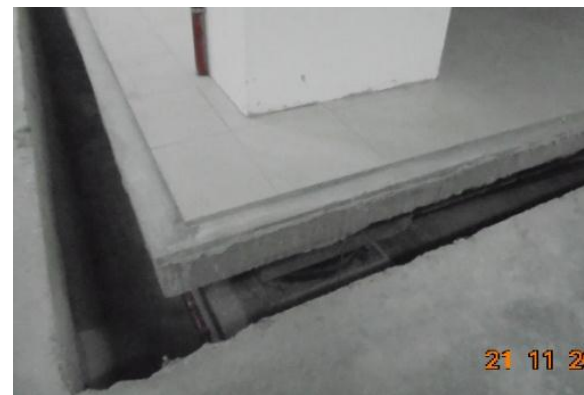

b)

Figura 9 a) Aisladores se encuentra sobre columna de subsuelo; b) Aisladores del bloque de ascensor y gradas se halla sobre los plintos.

En la Tabla 1 se resumen las estructuras con aislación sísmica hasta el presente año 2016 en el Ecuador.

Tabla 1 Estructuras con aislación sísmica en el Ecuador, hasta el año 2016

\begin{tabular}{|c|c|c|}
\hline No. & Estructuras & Bloques Estructurales \\
\hline 1 & $\begin{array}{c}\text { Centro de Investigación y Posgrado de } \\
\text { la UFA-ESPE }\end{array}$ & 5 \\
\hline 2 & Edificio Sede de UNASUR & 1 \\
\hline 3 & Sky Building & 1 \\
\hline 4 & Aeropuerto Internacional Mariscal Sucre & 1 \\
\hline 5 & Puente "Los Caras" & 1 \\
\hline 6 & Puente de Esmeraldas Norte & 1 \\
\hline 7 & Puente de Esmeraldas Norte1 & 1 \\
\hline 8 & Puente de Esmeraldas Sur & 1 \\
\hline 9 & Puente sobre el Río Chiche & 1 \\
\hline
\end{tabular}

En el diseño de estructuras con aislación sísmica se requiere que el sistema de aislación sea capaz de responder las deformaciones y cargas correspondiente al sismo máximo considerado, MCE. Con el objetivo, que la superestructura se mantenga en el rango elástico durante el sismo. Por lo tanto, la verificación del desempeño para el MCE se debe realizar analítica y experimentalmente. El criterio de esta verificación se basa en exhibir el peor escenario sísmico posible. Por tal motivo el presente estudio, busca realizar la verificación analítica del desempeño del aislador de Triple Péndulo de Fricción con el modelo en la fase II de McVitty y Constantinou (2015) y comparar con el modelo bilineal mediante un aplicativo en el software Matlab.

\section{MODELO DE MCVITTY Y CONSTANTINOU (2015)}

El aislador Triple Péndulo de Fricción (Triple FP) tiene un comportamiento no lineal y complejo. A medida que el desplazamiento aumenta, hay múltiples cambios en la rigidez. Típicamente a bajos niveles de fuerza / desplazamiento el sistema es muy rígido, en comparación a un sismo de diseño (DBE) en el que hay una menor rigidez y un aumento de la amortiguación, y el comportamiento también difiere a un sismo máximo considerado (MCE) donde hay una mayor rigidez para controlar los desplazamientos. Esto da lugar a un sistema adaptativo que puede ser optimizado para diferentes niveles de rendimiento. 
En Fenz y Constantinou (2008, 2008) y Fadi y Constantinou (2010) presentaron un modelo de cinco fases, en base a los 12 parámetros geométricos y cuatro coeficientes de roce que definen el comportamiento fuerza-desplazamiento del aislador triple FP. En este modelo de McVitty y Constantinou (2015), se reduce el número de parámetros geométricos a 6 y los coeficientes de roce a 2, observar Figura 10, a).

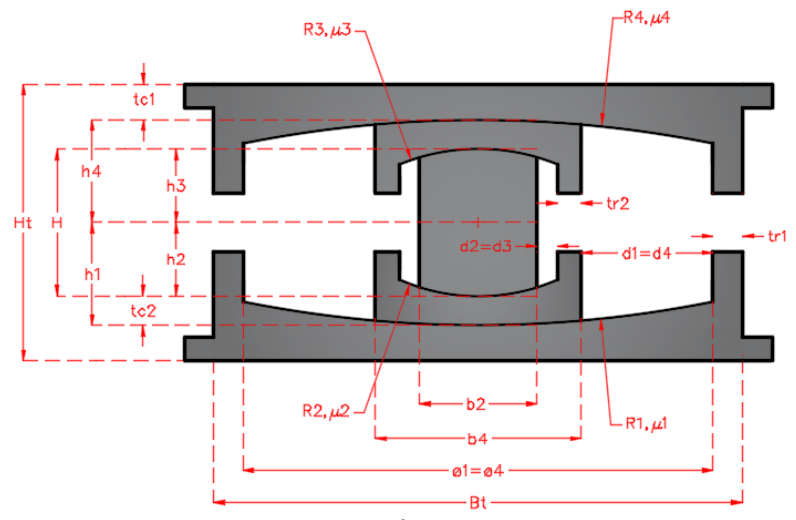

a)

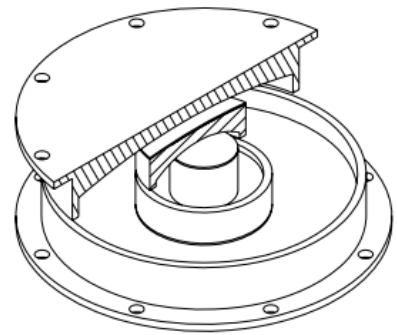

b)

Figura 10 a) Sección transversal y b) Vista en corte del aislador de Triple FP Fuente: McVitty y Constantinou (2015)

Reducción de parámetros:

- $R_{1}=R_{4} \gg R_{2}=R_{3}$

- $\mu_{2}=\mu_{3}<\mu_{1}=\mu_{4}$

- $d_{1}=d_{4}$ y $d_{2}=d_{3}$

- $h_{1}=h_{4}$ y $h_{2}=h_{3}$

$$
\begin{aligned}
& R_{\text {ieff }}=R_{i}-h_{i} \\
& d_{i}^{*}=\frac{R_{\text {ieff }}}{R_{i}} d_{i}
\end{aligned}
$$

Donde $R_{i}$ es el radio de curvatura; $h_{i}$ y $d_{i}$ es la altura y el desplazamiento presentado en la Figura $10 ; R_{\text {ieff }}$ es el radio de curvatura efectivo; $d_{i}^{*}$ es la capacidad de desplazamiento; $\mu_{i}$ es el coeficiente de fricción. El subíndice $i$, varia de 1 a 4 debido que existen 4 superficies.

Se adopta estos parámetros al comportamiento Fuerza-Desplazamiento y se reduce a tres régimen.

\section{Régimen I}

El desplazamiento se da solamente en las placas 2 y 3 .

$$
\begin{gathered}
0 \leq u \leq u^{*} \\
u^{*}=2\left(\mu_{1}-\mu_{2}\right) R_{2 e f f}
\end{gathered}
$$




$$
F=\left(\frac{W}{2 R_{2 e f f}}\right) u+\mu_{2} W
$$

Donde $u$ es el desplazamiento lateral del aislador; $u^{*}$ máximo desplazamiento en la fase I; $F$ es la fuerza lateral aplicada; $W$ es el peso que gravita sobre el aislador.

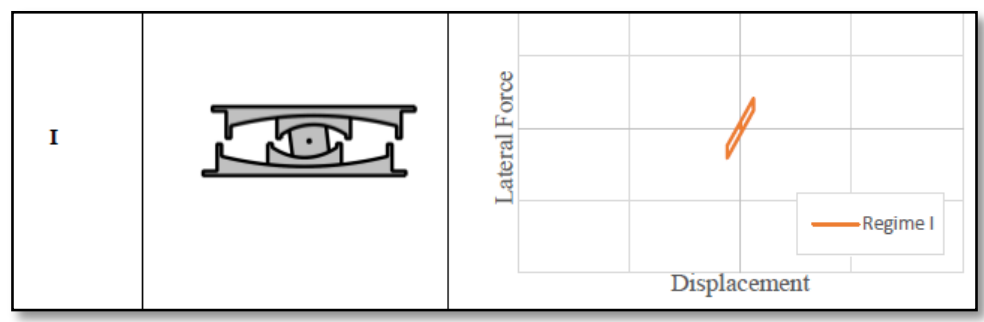

Figura 11 Régimen I

Fuente: McVitty y Constantinou (2015)

\section{Régimen II}

El movimiento en el aislador interior llega a los topes y empieza a desplazarse en las superficies 1 y 4 .

$$
\begin{gathered}
u^{*} \leq u \leq u^{* *} \\
u^{* *}=u^{*}+2 d_{1}^{*} \\
F=\left(\frac{W}{2 R_{1 e f f}}\right)\left(u-u^{*}\right)+\mu_{1} W
\end{gathered}
$$

Donde $u$ es el desplazamiento lateral del aislador; $u^{*}$ máximo desplazamiento en la fase I; $u^{* *}$ máximo desplazamiento en la fase II; $F$ es la fuerza lateral aplicada; $W$ es el peso que gravita sobre el aislador.

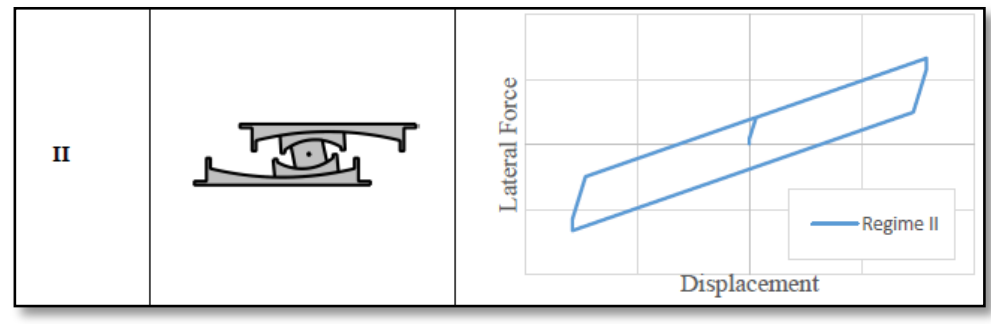

Figura 12 Régimen II

Fuente: McVitty y Constantinou (2015)

\section{Régimen III}

Este régimen se presenta cuando el sismo es extremadamente fuerte y el aislador interior se topa con los topes exteriores, en estas condiciones empieza a deslizarse el aislador interior en las superficies 2 y 3.

$$
u^{* *} \leq u \leq u_{c a p}
$$




$$
\begin{gathered}
u_{\text {cap }}=2 d_{1}{ }^{*}+2 d_{2}{ }^{*} \\
F=\left(\frac{W}{2 R_{2 e f f}}\right)\left(u-u^{* *}\right)+\left(\frac{W}{2 R_{1 e f f}}\right)\left(u^{* *}-u^{*}\right)+\mu_{1} W
\end{gathered}
$$

Donde $u$ es el desplazamiento lateral del aislador; $u^{* *}$ máximo desplazamiento en la fase II; $u_{c a p}$ máximo desplazamiento en la fase III; $F$ es la fuerza lateral aplicada; $W$ es el peso que gravita sobre el aislador.

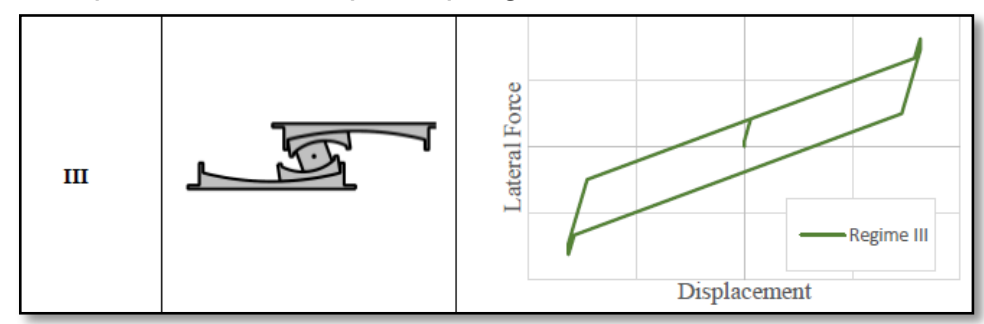

Figura 13 Régimen III

Fuente: McVitty y Constantinou (2015)

El análisis sísmico de edificaciones con aislación sísmica requiere un modelo matemático del comportamiento fuerza-desplazamiento del aislador. De tal manera, para validar el modelo tri-lineal propuesto por McVitty y Constantinou (2015), el aislador no alcanza el Régimen III para desplazamientos MCE. En conclusión, el diagrama fuerza-desplazamiento puede ser modelado en el Régimen Il como se muestra en la Figura 14. La fuerza en el desplazamiento cero viene dada por:

$$
\mu W=\left[\mu_{1}-\left(\mu_{1}-\mu_{2}\right) \frac{R_{2 e f f}}{R_{1 e f f}}\right] W
$$

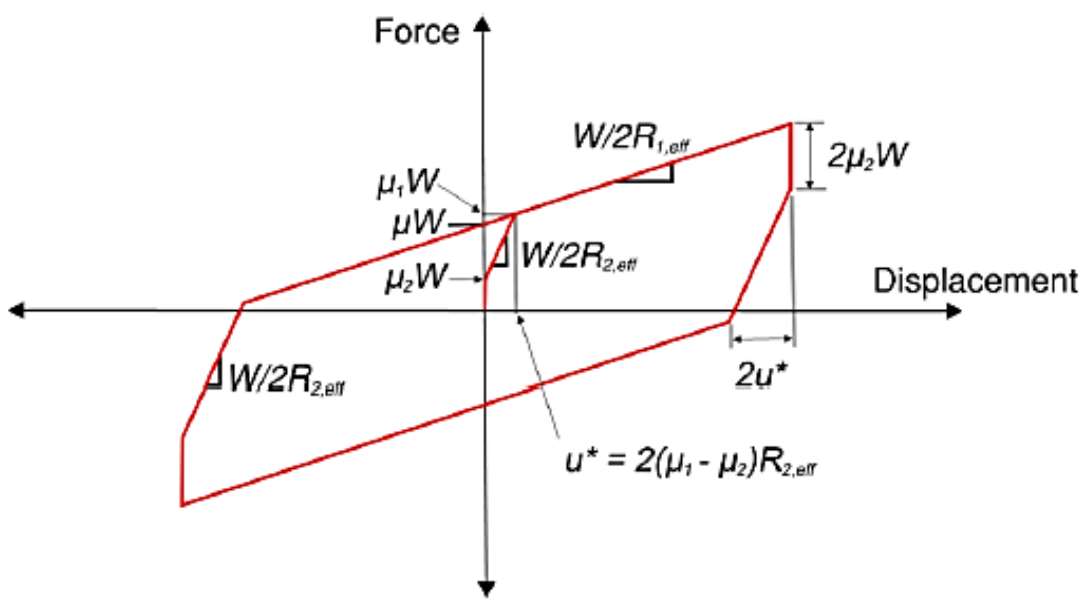

Figura 14 Modelo Tri-lineal en el Régimen II Fuente: McVitty y Constantinou (2015) 


\section{DESCRIPCIÓN DEL APLICATIVO EN MATLAB}

El aplicativo realiza un análisis no lineal de un grado de libertad para la verificación analítica del desempeño del aislador de Triple Péndulo de Fricción. Este análisis se plantea con un modelo tri-lineal propuesto por McVitty y Constantinou (2015).

El punto de fluencia del modelo tri-lineal, se halla con las ecuaciones que definen la fuerza y desplazamiento en el régimen I; y el punto último se encuentra con la ecuación que determina la fuerza y desplazamiento para el régimen II. Es un modelo conservador ya que se tiene en la realidad como punto último las ecuaciones del régimen III.

En este apartado se describe brevemente los aspectos fundamentales del análisis no lineal, para el modelo constitutivo tri-lineal.
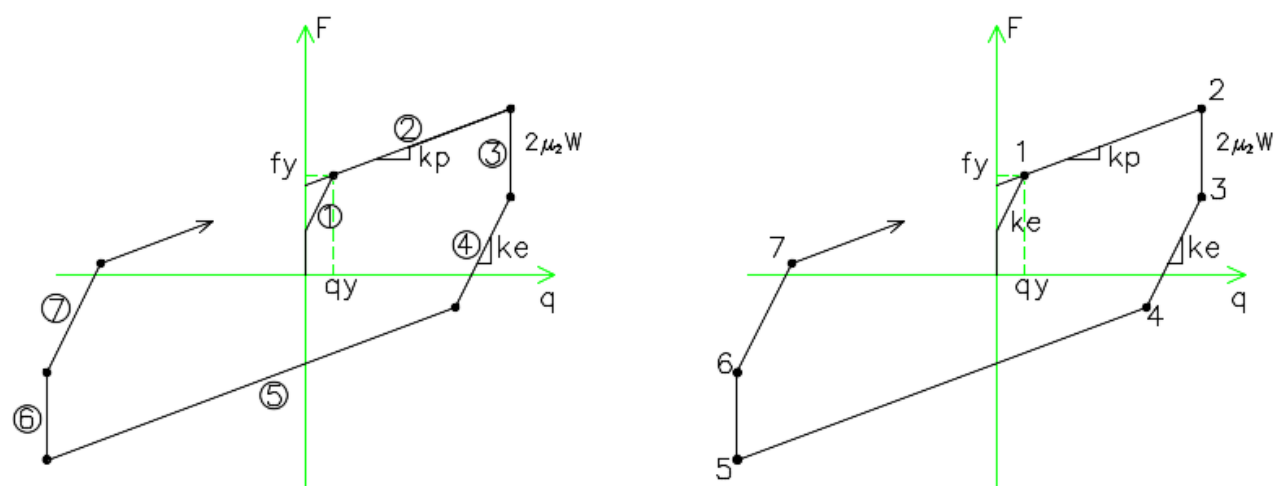

Figura 15 Identificación de las ramas y puntos de quiebre del Modelo tri-lineal

A la izquierda de la Figura 15 se definen las 7 ramas que considera el modelo, en la rama 1 trabaja exclusivamente en el rango elástico con una rigidez $K e$ que es la pendiente de esta recta, cuando se supera la fuerza de fluencia fy se ingresa a la rama 2 denominada post fluencia cuya pendiente es la rigidez $\mathrm{k} p$. En la rama dos se va controlando la velocidad, cuando esta llega a cero se alcanza el punto de control 2, que se indica a la derecha de la Figura 15; en la rama 3 se queda un momento en reposo hasta que la fuerza lateral supera a la fuerza de fricción, $2 \mu_{2} W$; ingresando a la rama de descarga definida por 4 con una rigidez $\mathrm{Ke}$. La rama 5 es similar a la rama 2, la rama 6 a la rama 3 y la rama 7 a la rama 4 pero actuando el sismo en sentido contrario. En análisis no lineal, es muy importante la determinación de los puntos de quiebre, en forma exacta y para lograr este objetivo se disminuye el incremento de tiempo $\Delta t$. Revisar Aguiar (2012) y Freire y Ortíz (2014).

\subsection{Método $\beta$ de newmark}

El marco teórico del método $\beta$ de Newmark, se halla descrito en Aguiar (2012) y Freire y Ortíz (2014). Aquí se presenta el procedimiento computacional para el análisis no lineal. Partiendo de que inicialmente se halla en la rama 1; en este caso y al inicio del programa para $t=0$, las condiciones iniciales son nulas. Con esta introducción el procedimiento de cálculo es el siguiente. 
i. Definir los datos del aislador y acelerograma:

Geometría del aislador; Coeficientes de Fricción;

$m=$ masa del aislador + masa actuante sobre el aislador $; W=$ peso total;

$g=$ Acelerograma $; p=g * m$

ii. Se determina el caso especial que se va a utilizar.

$\rightarrow$ Método de la aceleración constante, $\gamma=1 / 2$ y $\beta=1 / 4$

$\rightarrow$ Método de la aceleración lineal, $\gamma=1 / 2$ y $\beta=1 / 6$

$\rightarrow$ Método de la aceleración escalonada, $\gamma=1 / 2$ y $\beta=1 / 8$

iii. Se define las Condiciones iniciales:

$$
q_{0}=0 \mathrm{~m} \quad \dot{q}_{0}=0 \mathrm{~m} / \mathrm{s} \quad \ddot{q}_{0}=0 \mathrm{~m} / \mathrm{s}^{2}
$$

Donde $q_{i}$ es el desplazamiento; $\dot{q}_{i}$ es la velocidad; $\ddot{q}_{i}$ es la aceleración.

Cálculos que se realizan para cada iteración i:

iv. Se determina el intervalo de tiempo $\Delta t$ :

$$
\Delta t=t_{i+1}-t_{i}
$$

v. Se calcula el amortiguamiento:

Los valores de $k_{i}$ depende de la rama en que se encuentra, observar Figura 15 y $\xi$ se detalla en el literal 3.2.

$$
c=2 \xi \sqrt{m * k_{i}}
$$

Donde $k_{i}$ es la rigidez elástica o plástica; $\xi$ es el factor de amortiguamiento; $c$ es el amortiguamiento para un sistema de un grado de libertad.

vi. Se obtiene la rigidez equivalente y el intervalo de carga:

$$
\begin{gathered}
a=\frac{1}{\beta * \Delta t} * m+\frac{\gamma}{\beta} * c \\
b=\frac{1}{2 * \beta} * m+\Delta t *\left(\frac{\gamma}{2 \beta}-1\right) * c \\
\hat{k}_{l}=k_{i}+\frac{\gamma}{\beta * \Delta t} * c+\frac{1}{\beta * \Delta t^{2}} * m \\
\Delta \hat{p}_{i}=\Delta p_{i}+a \dot{q}_{l}+b \ddot{q}_{l}
\end{gathered}
$$

Donde $\hat{k}_{l}$ es la rigidez equivalente; $\Delta \hat{p}_{i}$ es el intervalo de carga.

vii. Se calcula el intervalo de desplazamiento y velocidad: 


$$
\begin{gathered}
\Delta q_{i}=\frac{\Delta \hat{p}_{i}}{\widehat{k}_{l}} \\
\Delta \dot{q}_{\iota}=\frac{\gamma}{\beta \Delta t} \Delta q_{i}-\frac{\gamma}{\beta} \dot{q}_{\imath}+\Delta t *\left(1-\frac{\gamma}{2 \beta}\right) \ddot{q}_{\imath}
\end{gathered}
$$

viii. Finalmente se obtiene la respuesta de desplazamiento, velocidad, la fuerza lateral y aceleración del aislador en cada instante de tiempo:

$$
\begin{aligned}
& q_{i+1}=\Delta q_{i}+q_{i} \\
& \dot{q}_{i+1}=\Delta \dot{q}+\dot{q}_{i}
\end{aligned}
$$

ix. La aceleración se calcula preferible mediante la ecuación diferencial de movimiento:

$$
\ddot{q}_{i+1}=\frac{p_{i+1}-c * \dot{q}_{i+1}-(f s)_{i+1}}{m}
$$

Se destaca que:

$$
(f s)_{i+1}=(f s)_{i}+k_{i} \Delta q_{i}
$$

Donde $f s$ es la fuerza lateral asociada a la rigidez, que va cambiando de acuerdo a la rama en que se encuentra el modelo.

\subsection{Factor de amortiguamiento (६)}

En la fase I el aislador empieza con un factor de amortiguamiento igual a 0.05 , debido que el aislador empieza trabajando con una rigidez alta y un amortiguamiento bajo. Cuando pasa a la fase II, se calcula en cada instante el factor de amortiguamiento con la siguiente ecuación:

$$
\xi=\frac{E_{D}}{4 \pi E_{S}}
$$

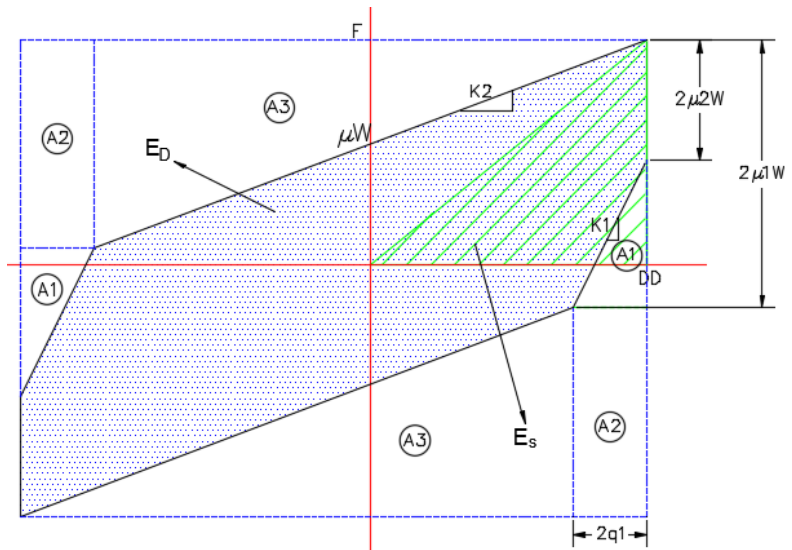

Figura 16 Cálculo de la Energía Disipada y Energía Elástica. 
En la Figura 16 el eje de las abscisas y ordenadas divide en 4 áreas iguales $(F * D D)$, y para encontrar la Energía Disipada por cada ciclo se resta las áreas externas, $A_{1}, A_{2}$ y $A_{3}$; como resultado la siguiente ecuación, se describe en Gutiérrez (2016):

$$
E_{D}=4(F * D D)-2 A_{1}-2 A_{2}-2 A_{3}
$$

Simplificando los términos:

$$
E_{D}=4 *\left(q_{1} * \mu_{2} * W-q_{1} * F+D D * 2 * \mu_{1} * W\right)
$$

Método de Cálculo

i. Se determina en cada instante de tiempo el desplazamiento, DD

ii. Con el desplazamiento se calcula su Fuerza, F.

$$
F=K_{2} * D D+\mu * W
$$

iii. Se calcula la Energía Disipada

$$
E_{D}=4 *\left(q_{1} * \mu_{2} * W-q_{1} * F+D D * 2 * \mu_{1} * W\right)
$$

iv. La Energía Elástica

$$
E_{s}=\frac{F * D D}{2}
$$

v. Zeda para cada instante.

$$
\xi=\frac{E_{D}}{4 \pi E_{S}}
$$

\section{ENSAYO DEL AISLADOR FPT8833/12-12/8-5}

El aislador FPT8833/12-12/8-5 del nuevo Centro de Investigación y Posgrado de la ESPE, fue ensayado bajo una función armónica que se presenta en la Figura 17. El periodo promedio de los bloques con aisladores sísmicos para el DBE es $T_{D}$ $=3 \mathrm{~s}$. Por lo tanto, para el ensayo del aislador se utilizó un frecuencia $\omega_{n}=2 \pi / T_{D}$.

a)

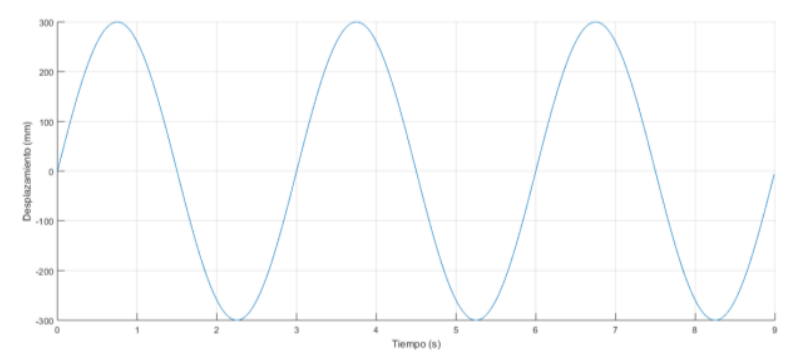


b)

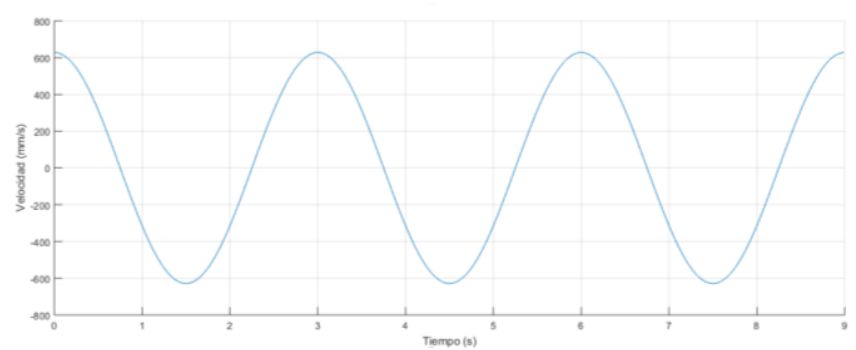

c)

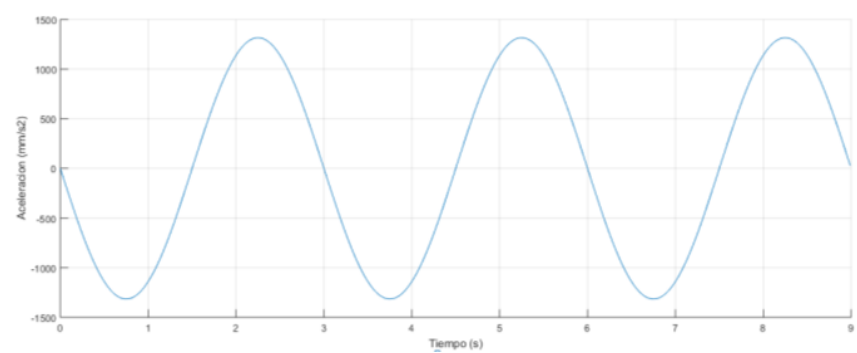

Figura 17 La función armónica a) Desplazamiento-Tiempo; b) Velocidad-Tiempo;

c) Aceleración-Tiempo

Se observa en la Figura 18 la constitutiva del ensayo experimental que es ajustada a un modelo matemático para determinar los coeficientes de fricción e imágenes del ensayo. En la Figura 19 se presenta la constitutiva analítica del modelo tri-lineal propuesto por McVitty y Constantinou (2015) aplicado la misma carga del ensayo experimental en el programa de Matlab.

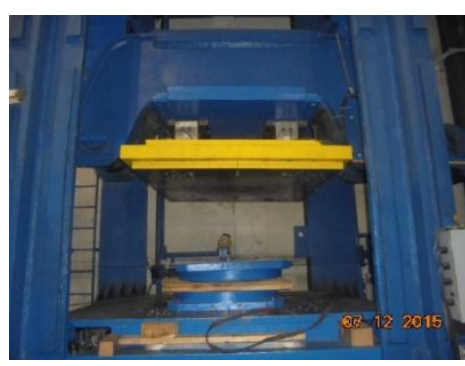

a)

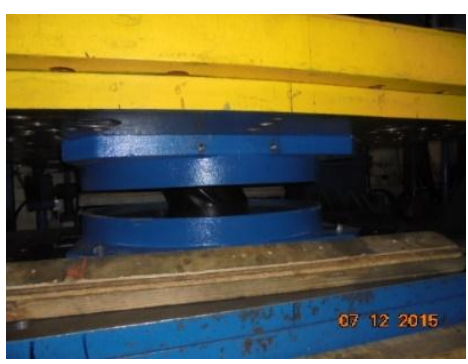

b)

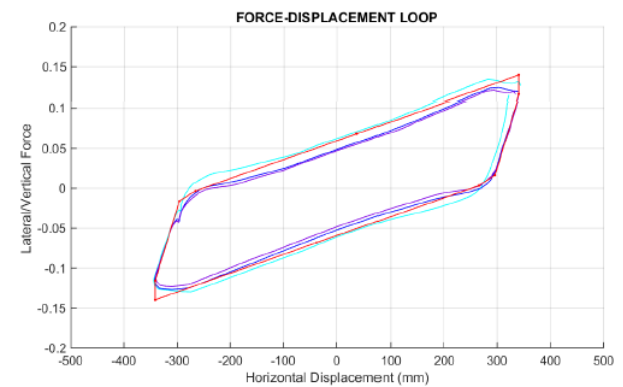

c)

Figura 18 a) Instalación del aislador en el ensayo experimental; b) Aplicación de la carga al aislador c) Constitutiva ajustada del ensayo experimental bajo tres ciclos de carga.

Fuente: EPS 


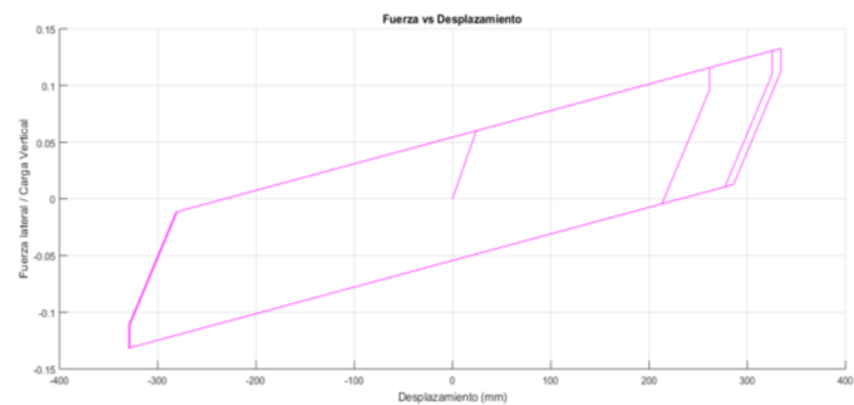

Figura 19 Constitutiva obtenida en este estudio con el modelo tri-lineal de McVitty y Constantinou (2015)

En el ensayo experimental empieza con una fuerza en el desplazamiento igual a cero, para obtener en los 3 ciclos desplazamientos semejantes. En cambio, en el aplicativo de Matlab se observa que en el ciclo 1 se obtiene un desplazamiento menor porque parte de la fase 1 y los dos ciclos siguientes dan el desplazamiento final.

El desplazamiento promedio del ensayo experimental en los 3 ciclos es de $342.3 \mathrm{~mm}$. En cambio, mediante el aplicativo en Matlab se obtuvo un desplazamiento de $334.3 \mathrm{~mm}$. Este dato permite verificar la programación del aplicativo del modelo matemático que plantea McVitty y Constantinou (2015) y se lo puede utilizar para realizar un análisis no linear respuesta en el tiempo del aislador de Triple Péndulo de Fricción en un sistema de 1 grado de libertad.

\subsection{Coeficientes de Fricción}

Para determinar los coeficientes de fricción del ensayo experimental se ajusta las gráficas a un modelo matemático, en este estudio se va explicar mediante el modelo de la fase II de McVitty y Constantinou (2015). A continuación se determina el proceso de cálculo:
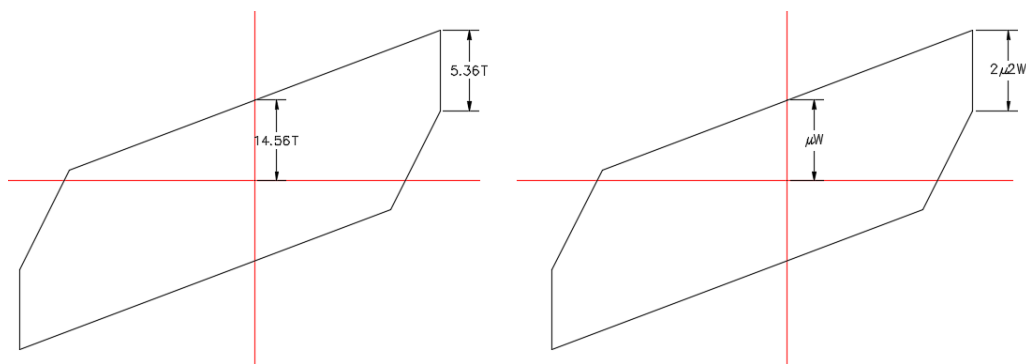

Figura 20 Modelo ajustado del ensayo experimental para determinar los coeficientes de fricción

Del modelo ajustado se extrae los dos valores que se muestra en la Figura 20 a la izquierda y se iguala con el modelo de la fase II de McVitty y Constantinou (2015). Primero se obtiene $\mu_{2}\left(5.36=2 \mu_{2} \mathrm{~W}\right)$ y para $\mu 1$ se despeja del valor de la fuerza en el desplazamiento cero $(14.56=\mu \mathrm{W})$, se conoce que: 


$$
\mu W=\left[\mu_{1}-\left(\mu_{1}-\mu_{2}\right) \frac{R_{2 e f f}}{R_{1 e f f}}\right] W
$$

\section{PELIGROSIDAD SÍSMICA Y ESPECTROS DE ANÁLISIS}

Quito se halla sobre cuatro fallas geológicas inversas, denominadas: Puengasi, llumbisi-La Bota, Carcelén el Inca y Bellavista-Catequilla, cuyas trazas y planos de falla se presentan en la Figura 21.
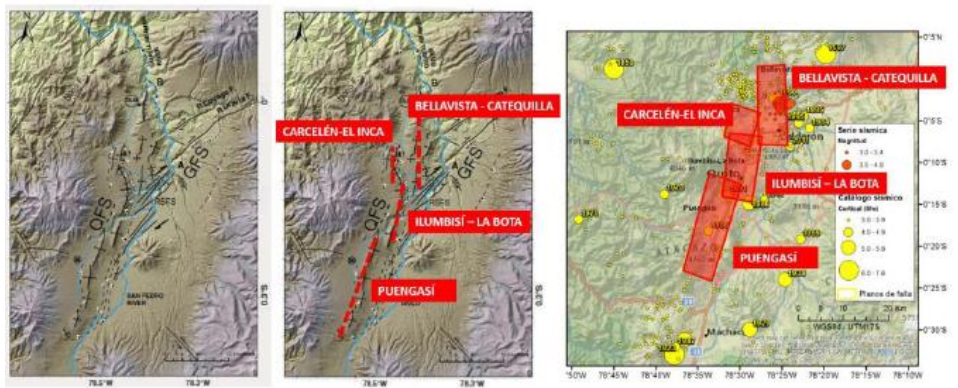

Figura 21 Modelos de Fallas ciegas de Quito, trazas y planos de falla.

Fuente: Rivas et al. (2014)

En el estudio de "Espectros de control para la ciudad de Quito-Ecuador" las mayores ordenadas espectrales para las zonas:

- Sur, centro sur y centro de Quito: se hallaron ante un sismo de magnitud 6.4 registrado en el centro del plano de falla del segmento de Puengasí.

- Centro norte y norte de Quito: se hallaron ante un sismo de magnitud 6.2, en la falla de llumbisí-La Bota.

La falla de Puengasí tiene un periodo de recurrencia de 1224 y 2190 años para que se produzca un sismo $\mathrm{Mw}=6.4$, valores hallados con modelo de Gutenberg y Richter Modificado. Rivas et al. (2014).

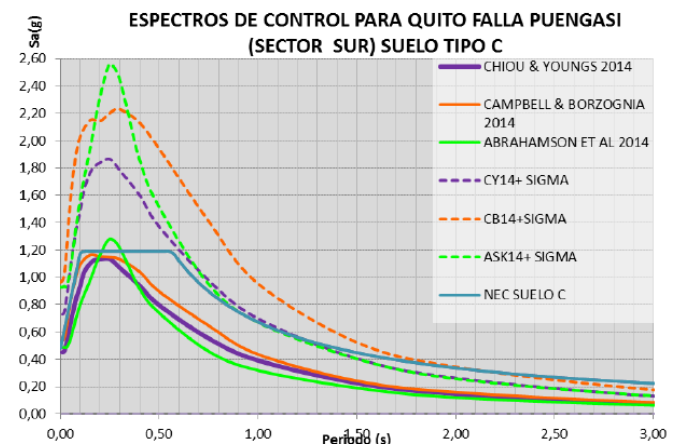

Figura 22 Espectros encontrados en perfil tipo C, para el sector Sur de Quito. Fuente: Aguiar (2015)

En la Figura 22 se presenta los espectros de análisis para la falla de Puengasí medidos en un perfil de suelo tipo "C" en el Sur de Quito, para niveles de 
confianza del 50\% (línea continua), 84\% (línea discontinua) y el espectro hallado con la NEC-15, el DBE.

En el período de 1 segundo, los espectros hallados con un nivel de confianza del $84 \%$ superan la aceleración espectral encontrada con el NEC-15. Por tal motivo, para encontrar el sismo MCE a partir del sismo DBE se busca un factor, que es la división hallada con el modelo de Campbell y Borzognia (2014) (línea naranja entrecortada) para la aceleración del NEC-15 y su valor es de 1.4.

\subsection{Acelerogramas seleccionados}

Los registros que se utilizarán deben asemejarse a las características geológicas de Quito, con las siguientes consideraciones que proponen Aguiar y Morales (2016):

- Los registros son de fallas cercanas a las fallas.

- Los registros del sismo de Gazly de 1976, provienen de una región tipo "backward-directivity". Los restantes registros son de regiones "forwarddirectivity".

- La magnitud de los registros se encuentra entre 6.7 y 7.1 .

- Las distancias a la fuente Rrup se encuentran entre 3 y $12 \mathrm{~km}$,

- Los registros fueron obtenidos en suelos tipo "C" y "D"

- Los registros de Gazli de 1976 y el de Loma Prieta de 1989, son considerados como registros estándar con alto contenido de frecuencia.

- Los sismos de Northridge de 1994 y Kobe de 1995 son sismos impulsivos y son considerados por su cercanía a la falla.

- Se incluye el sismo de Turquía de 1999 porque fue altamente destructivo.

Tabla 2 Registro de sismos seleccionados

\begin{tabular}{|c|c|c|c|c|c|}
\hline Código & Nombre del Sismo & Estación & $\mathbf{M}_{\mathbf{w}}$ & $\mathbf{R}(\mathbf{K m})$ & $\begin{array}{c}\text { Tipo de } \\
\text { Suelo }\end{array}$ \\
\hline EQ1 & 1976 Gazli, USSR & Karakyr & 6.80 & 5.46 & $\mathrm{C}$ \\
\hline EQ2 & 1989 Loma Prieta & LGPC & 6.93 & 3.88 & $\mathrm{C}$ \\
\hline EQ3 & 1989 Loma Prieta & $\begin{array}{c}\text { Saratoga-W } \\
\text { Valley Coll }\end{array}$ & 6.93 & 9.31 & $\mathrm{C}$ \\
\hline EQ4 & 1994 Northridge & $\begin{array}{c}\text { Jensen Filter } \\
\text { Plan }\end{array}$ & 6.69 & 5.43 & $\mathrm{C}$ \\
\hline EQ5 & 1994 Northridge & $\begin{array}{c}\text { Sylmar-Coverter } \\
\text { Sta. East }\end{array}$ & 6.69 & 5.19 & $\mathrm{C}$ \\
\hline EQ6 & 1995 Kobe, Japón & Takarazuka & 6.90 & 3.00 & $\mathrm{D}$ \\
\hline EQ7 & $\begin{array}{c}\text { 1999 Duzce, } \\
\text { Turquía }\end{array}$ & Bolu & 7.14 & 12.41 & D \\
\hline
\end{tabular}

\subsection{Espectros de respuesta elásticos}

El espectro de diseño denominado DBE, que corresponde al NEC-15 y el máximo considerado MCE (observar la Figura 23) que se obtiene al multiplicar el DBE por 1.4, dicho factor se determinó en el apartado anterior. 
En la Figura 24 están los espectros obtenidos de cada acelerograma en la componente normal y paralela a la falla para un factor de amortiguamiento del $5 \%$; la línea roja es el promedio de todos los espectros, $50 \%$ de nivel de confianza.

Como se puede observar en la Figura 25 el espectro MCE está por encima del espectro promedio de los registros seleccionados, por lo tanto se debe escalar al MCE los registros para los periodos de interés.

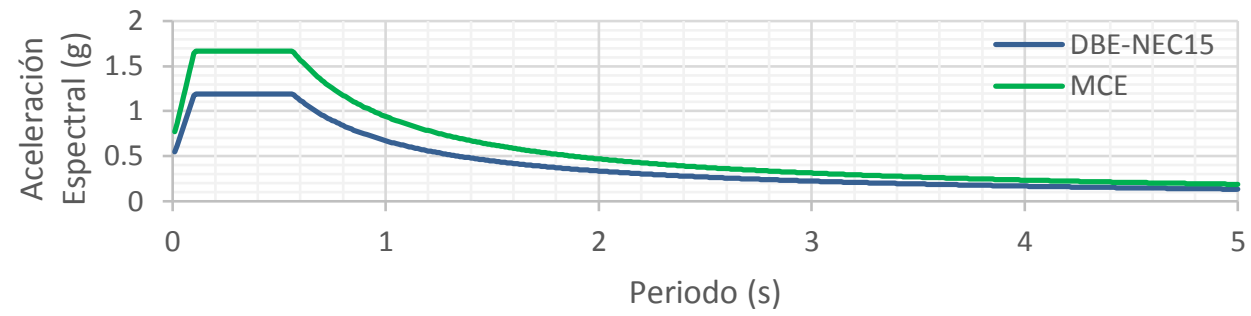

Figura 23 Espectros elásticos de Diseño DBE y máximos considerados MCE para Quito en un suelo tipo "C"

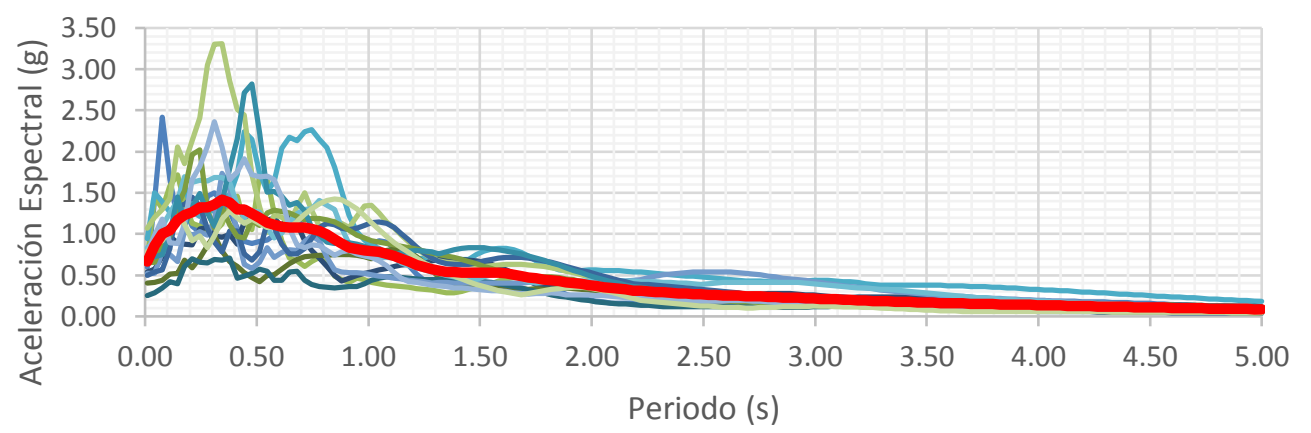

Figura 24 Espectros elásticos de los registros considerados para el Sur de Quito

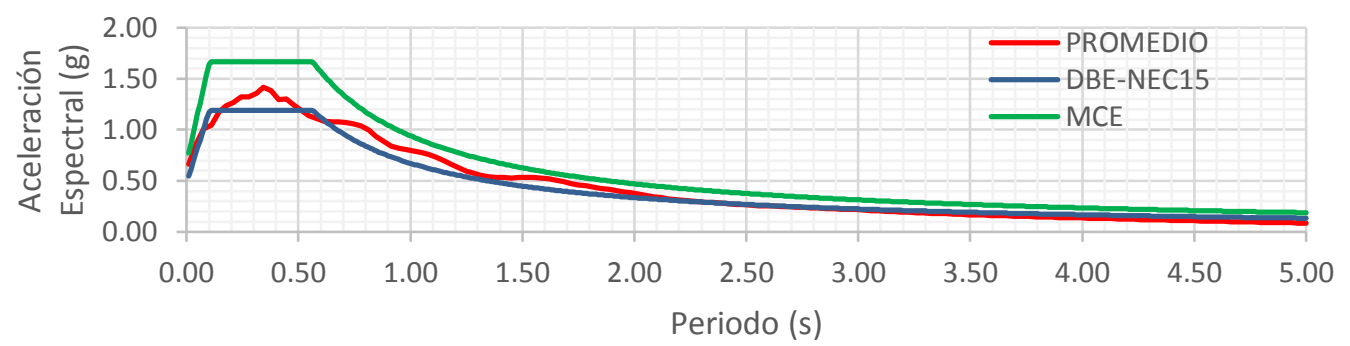

Figura 25 Espectros de diseño DBE, máximo considerado MCE y promedio de los registros seleccionados

\subsection{Factores de escalamiento}

Para el escalamiento de sismos el rango de los periodos es $0.5 \mathrm{~T}_{\mathrm{D}}$ a $1.25 \mathrm{~T} \mathrm{M}$. El periodo de la estructura para el DBE es $T_{D}$ y para el MCE es $T_{M}$. Para los cincos bloques estructurales de la UFA-ESPE el $T_{D}=3 \mathrm{~s}$ y el $T_{M}=3.4 \mathrm{~s}$. De tal manera el rango de escalonamiento es de 1.5 y 4.25. En la Tabla 3 se presenta el factor de escalonamiento para cada registro. 
Tabla 3 Factor de Escalonamiento mediante el método del Promedio de Pesos

Ponderados.

\begin{tabular}{|c|c|c|c|c|c|c|c|}
\hline \multicolumn{7}{|c|}{ FACTOR DE ESCALONAMIENTO } \\
\hline & EQ1 & EQ2 & EQ3 & EQ4 & EQ5 & EQ6 & EQ7 \\
\hline Sa MCE $(\mathrm{t}=1)$ & 0.941 & 0.941 & 0.941 & 0.941 & 0.941 & 0.941 & 0.941 \\
\hline Sa FN $(\mathrm{t}=1)$ & 0.815 & 0.873 & 0.710 & 0.512 & 0.732 & 0.933 & 0.729 \\
\hline Sa MCE $(\mathrm{t}=2)$ & 0.471 & 0.471 & 0.471 & 0.471 & 0.471 & 0.471 & 0.471 \\
\hline Sa FN $(\mathrm{t}=2)$ & 0.198 & 0.561 & 0.314 & 0.411 & 0.429 & 0.396 & 0.259 \\
\hline Sa MCE $(\mathrm{t}=3)$ & 0.314 & 0.314 & 0.314 & 0.314 & 0.314 & 0.314 & 0.314 \\
\hline Sa FN $(\mathrm{t}=3)$ & 0.161 & 0.436 & 0.176 & 0.421 & 0.394 & 0.148 & 0.146 \\
\hline Sa MCE $(\mathrm{t}=4)$ & 0.235 & 0.235 & 0.235 & 0.235 & 0.235 & 0.235 & 0.235 \\
\hline Sa FN $(\mathrm{t}=4)$ & 0.191 & 0.327 & 0.078 & 0.195 & 0.186 & 0.072 & 0.084 \\
\hline $\mathrm{Fj}$ & 1.375 & 0.869 & 1.461 & 1.128 & 1.084 & 1.144 & 1.527 \\
\hline $\mathrm{F} 1$ & 1.200 & 1.200 & 1.200 & 1.200 & 1.200 & 1.200 & 1.200 \\
\hline $\mathrm{F} 1 \mathrm{Fj}$ & 1.650 & 1.043 & 1.754 & 1.353 & 1.301 & 1.373 & 1.833 \\
\hline
\end{tabular}

En la Figura 26 se representa con línea roja el promedio de los registros multiplicados por el factor de escalonamiento respectivo, como resultado se tiene que las ordenadas estén sobre el MCE.

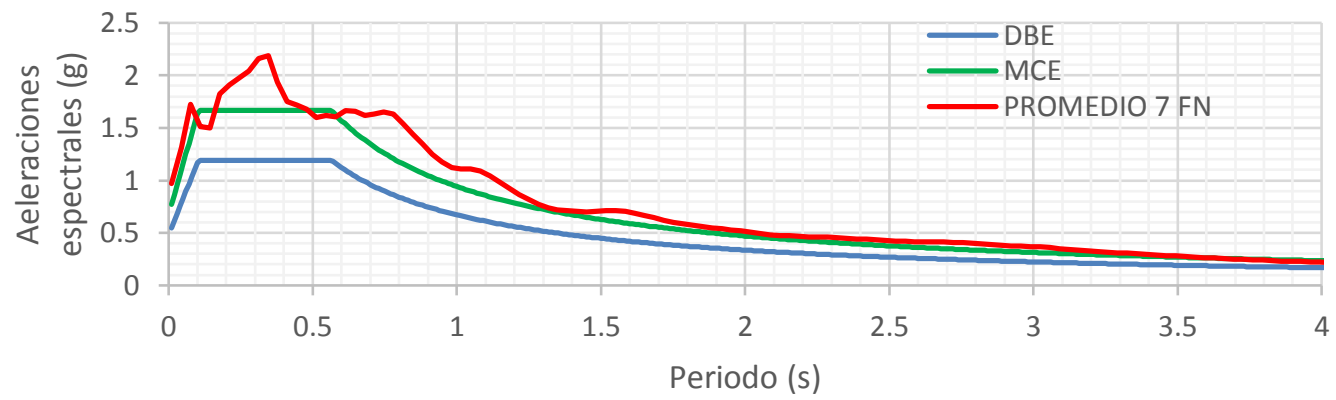

Figura 26 Espectro de Diseño DBE, máximo considerado MCE y promedio de los 7 sismos $\mathrm{FN}$

\section{DESEMPEÑO DEL AISLADOR FPT8833/12-12/8-5}

El aislador FPT8833/12-12/8-5 del nuevo Centro de Investigación y Posgrado de la ESPE se va a determinar su desempeño con el modelo tri-lineal de McVitty y Constantinou (2015) en el sector Sur de Quito para un suelo tipo "C" para comparar con los resultados obtenidos en Aguiar y Morales (2016). Esta comparación permite evaluar el modelo tri-lineal con respecto al bilineal, en la Figura 27 se presenta ambos modelos.

La geometría y coeficientes de fricción del aislador están descritos en Constantinou et al. (2016) 


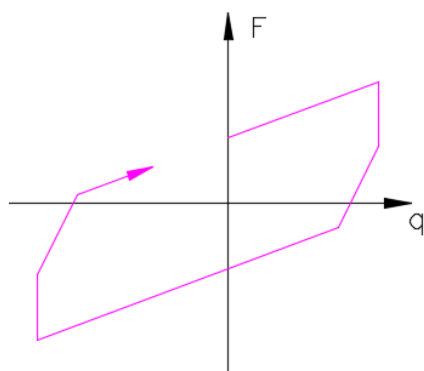

a)

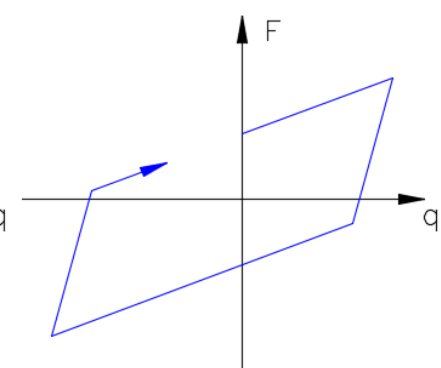

b)

Figura 27 Constitutivas utilizadas a) Modelo Tri-lineal; b) Modelo Bilineal

\subsection{Resultados}

Para determinar el desempeño del aislador 8833/12-12/8-5, se realizó mediante el modelo de tres fases de McVitty y Constantinou (2015). Los coeficientes de fricción se consideraron sus valores nominales y una carga de 268 Toneladas.

A los acelerogramas seleccionados se les multiplico por el factor de escalamiento respectivamente para encontrar la respuesta en el tiempo del aislador mediante el modelo tri-lineal (color magenta) y bilineal (color azul), estos resultados se presentan en la Figura 28.

Cabe indicar que el valor del $\xi$ se calcula en cada instante para simular el comportamiento no lineal y no trabajar únicamente con el छefectivo, como se lo realizó en el modelo bilineal.

Adicionalmente se presenta una gráfica comparativa del desplazamiento en el tiempo de cada registro mediante los dos modelos, para comparar sus desplazamientos, observar en la Figura 29.
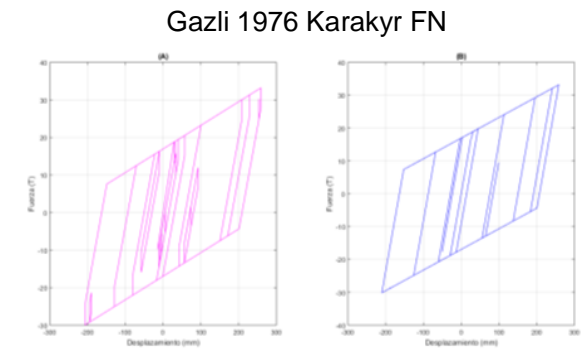

Loma Prieta 1989 Saratoga FN
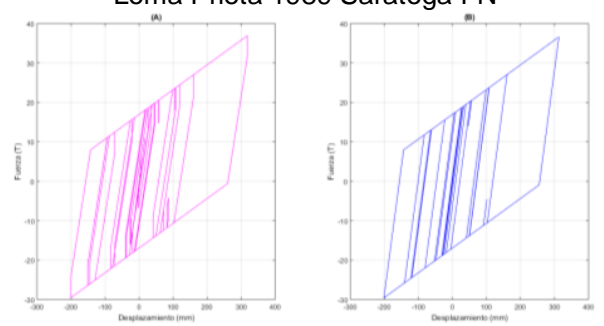

Loma Prieta 1989 LGPC FN
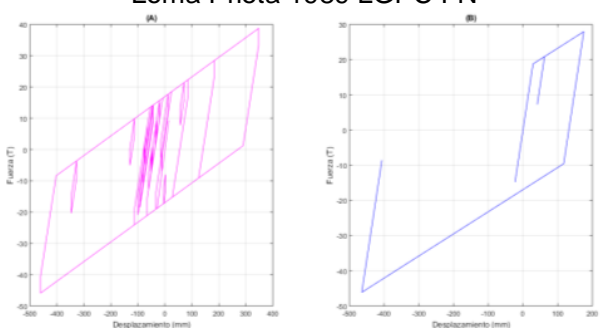

Northridge 1994 Jensen FN
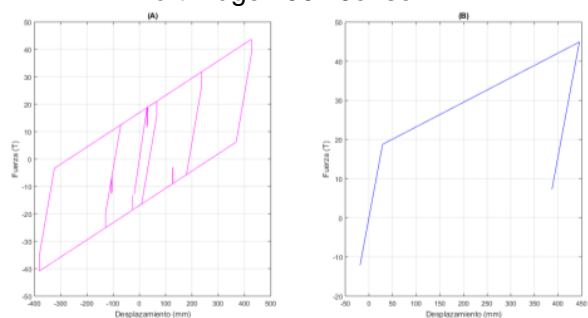

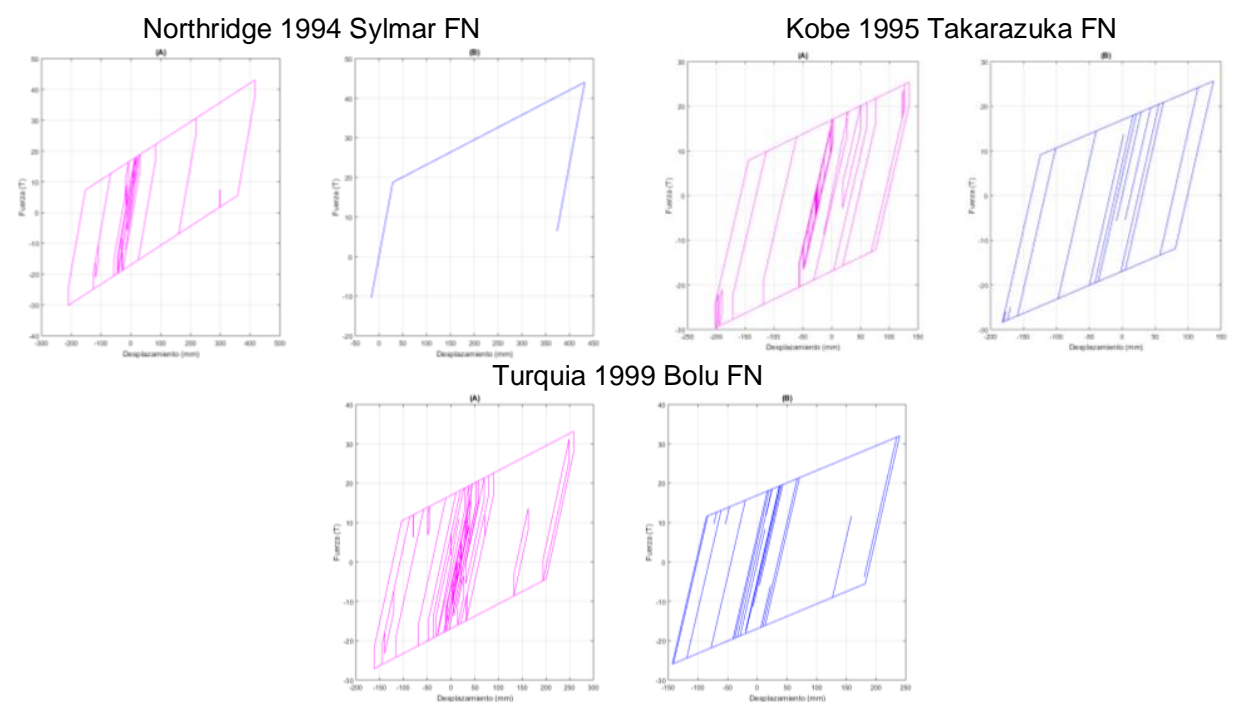

Figura 28 Respuesta en el Tiempo del aislador con los 7 registros FN mediante el comportamiento (A) Tri-lineal y (B) Bilineal.

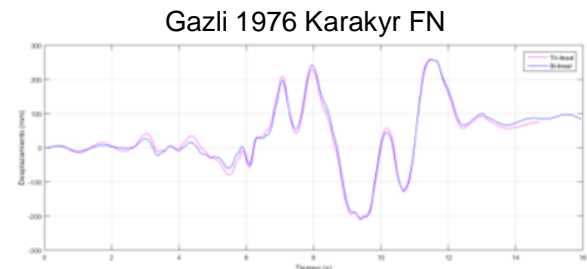

Loma Prieta 1989 Saratoga FN

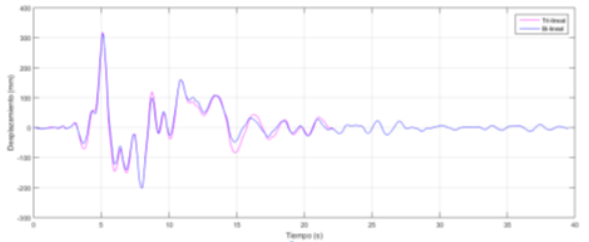

Northridge 1994 Sylmar FN

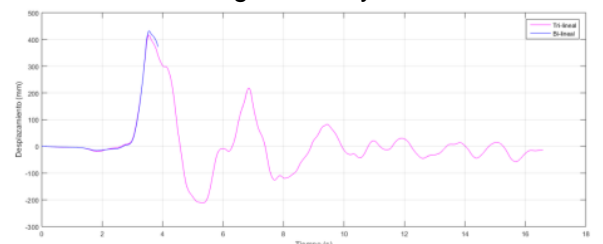

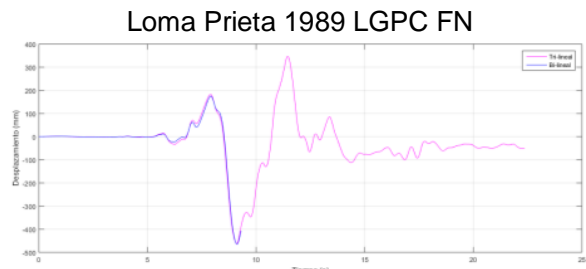

Northridge 1994 Jensen FN

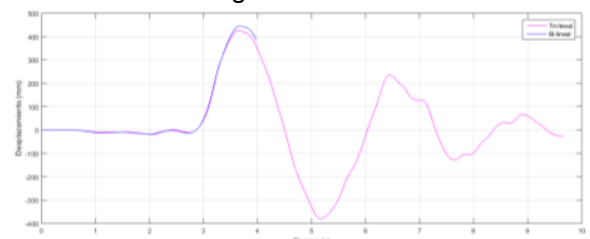

Kobe 1995 Takarazuka FN

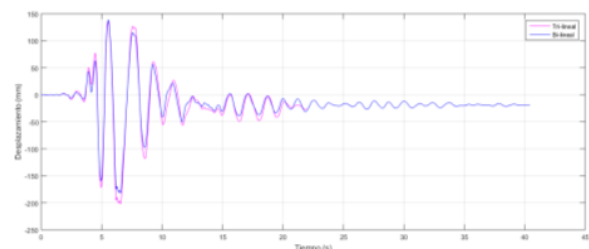

Turquia 1999 Bolu FN

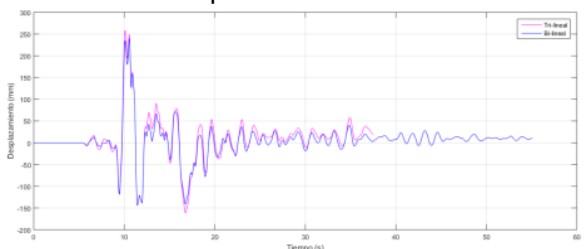

Figura 29 Gráfica comparativa del desplazamiento en el tiempo mediante el modelo tri-lineal y bilineal. 
Mediante el nuevo aplicativo en Matlab se observa mejores resultados porque el programa está ejecutándose mientras se tiene las mayores aceleraciones del registro y esto permite verificar analíticamente el desplazamiento máximo esperado. Pero se aprecia que deja de correr el programa cuando las aceleraciones tienden a cero debido que el aislador es un sistema adaptativo y para bajas condiciones de fuerzas es muy rígido.

En la Figura 29 se aprecia claramente que el desplazamiento en el tiempo tiene el mismo comportamiento en ambos modelos, que era el resultado que se esperaba para corroborar los modelos.

En la Tabla 4 está detallado los desplazamiento máximos esperados en cada sismo mediante los dos modelos y el mayor desplazamiento obtenido con el modelos tri-lineal es de $46 \mathrm{~cm}$ y con el bilineal es $46.4 \mathrm{~cm}$. El sismo de Loma Prieta es el que genera mayores desplazamientos. La capacidad de desplazamiento máximo del aislador en la fase II es $53.3 \mathrm{~cm}$, de tal manera se verifica analíticamente que el aislador FPT8833/12-12/8-5 trabaja perfectamente bajo las condiciones geológicas del sector Sur de Quito en un suelo tipo "C".

Tabla 4 Validación de los desplazamientos en la Fase II mediante el modelo Trilineal y Bilineal en los 7 registros.

\begin{tabular}{|c|c|c|c|c|c|}
\hline \multirow{2}{*}{ Código } & \multirow{2}{*}{$\begin{array}{l}\text { Nombre del } \\
\text { Sismo }\end{array}$} & \multirow{2}{*}{ Estación } & \multicolumn{2}{|c|}{$\begin{array}{l}\text { Desplazamiento } \\
\operatorname{Max}(\mathrm{mm})\end{array}$} & \multirow{2}{*}{$\begin{array}{c}\text { Diferencia } \\
\text { desplazamientos } \\
(\%)\end{array}$} \\
\hline & & & Tri-lineal & Bilineal & \\
\hline EQ1 & $\begin{array}{l}1976 \text { Gazli, } \\
\text { USSR }\end{array}$ & Karakyr & 258.9874 & 258.5895 & -0.15 \\
\hline EQ2 & $\begin{array}{l}1989 \text { Loma } \\
\text { Prieta }\end{array}$ & LGPC & 460.7535 & 464.3997 & 0.79 \\
\hline EQ3 & $\begin{array}{l}1989 \text { Loma } \\
\text { Prieta }\end{array}$ & $\begin{array}{l}\text { Saratoga-W } \\
\text { Valley Coll }\end{array}$ & 317.7102 & 312.78 & -1.55 \\
\hline EQ4 & $\begin{array}{c}1994 \\
\text { Northridge }\end{array}$ & $\begin{array}{l}\text { Jensen } \\
\text { Filter Plan }\end{array}$ & 426.3481 & 445.1043 & 4.40 \\
\hline EQ5 & $\begin{array}{c}1994 \\
\text { Northridge }\end{array}$ & $\begin{array}{l}\text { Sylmar- } \\
\text { Coverter } \\
\text { Sta. East }\end{array}$ & 415.9048 & 431.5528 & 3.76 \\
\hline EQ6 & $\begin{array}{l}1995 \text { Kobe, } \\
\text { Japón }\end{array}$ & Takarazuka & 134.534 & 138.6055 & 3.03 \\
\hline EQ7 & $\begin{array}{l}1999 \text { Duzce, } \\
\text { Turquía }\end{array}$ & Bolu & 258.1822 & 239.668 & -7.17 \\
\hline
\end{tabular}

\section{CONCLUSIONES}

El comportamiento del aislador de Triple Péndulo de Fricción es no lineal y complejo. Con el modelo matemático que propone McVitty y Constantinou (2015) permite verificar analíticamente el desempeño del aislador, como se pudo evidenciar en el ensayo experimental se obtuvo un desplazamiento de $34.2 \mathrm{~cm}$, y analíticamente un desplazamiento de $33.4 \mathrm{~cm}$ equivalente a un error del $3 \%$. Con este resultado se verifica la utilización para un análisis sísmico el modelo tri-lineal de la fase II de McVitty y Constantinou (2015). 
Comparar los resultados del modelo tri-lineal y bilineal para el análisis no lineal de un sistema de un grado de libertad permite afirmar que ambos modelos son útiles para determinar el desempeño del aislador. En la Tabla 4 se presenta los desplazamientos máximos para cada registro y la diferencia en desplazamiento entre ambos modelo, la más alta se obtuvo en el sismo de Duzce 1999 del 7\%, esta cifra más el $3 \%$ de error obtenida en el ensayo experimental es $10 \%$. Por lo tanto, para un prediseño si se recomienda trabajar con el modelo bilineal pero para el diseño final se debería trabajar con el modelo tri-lineal de la fase II.

Al aislador FPT8833/12-12/8-5 utilizado en el nuevo Centro de Investigación y Posgrado de la UFA-ESPE se determinó su desempeño bajo la demanda sísmica encontrada en el presente estudio para el sector Sur de Quito en un suelo tipo "C", con un desplazamiento máximo de $46 \mathrm{~cm}$ en el sismo de Loma Prieta 1989, previamente escalado al espectro máximo considerado. Se concluye que el uso del aislador FPT8833/12-12/8-5 se comporta favorablemente porque la capacidad de desplazamiento es mayor a la demanda sísmica calculada.

\section{REFERENCIAS}

1. Aguiar, R. (2015). Espectros de control para la ciudad de Quito-Ecuador. Centro Internacional de Métodos Numéricos de Ingeniería CIMNE , Universidad Politécnica de Cataluña, Barcelona, España.

2. Aguiar, R., y Morales, E. (2016). Desempeño de Aislador FPT8833/12-12/5 empleado en la construcción del Centro de Investigaciones de UFA-ESPE mediante análisis no lineal. Revista Internacional de Ingeniería de Estructuras, 21(1), 79-104.

3. Constantinou, M., Aguiar, R., Morales, E., y Caiza, P. (2016). Desempeño de Aislador FPT8833/12-12/-5 en el Análisis Sísmico del Centro de Investigaciones y de Post Grado de la UFA-ESPE. Revista Internacional de Ingeniería de Estructuras, 21(1), 1-26.

4. Constantinou, M., Filiatrault, A., Kalpakidis, I., y Ecker Lay, R. (2011). LRFDBased Analysis and Design Procedures for Bridge Bearings and Seismic Isolators. Technical Report MCEER-11-0004, University at Buffalo, State University of New York.

5. Fadi, D., y Constantinou, M. (2010). Evaluation of simplified methods of analysis for structures with triple friction pendulum isolators. Earthquake Engineering and Structural Dynamics.

6. Fenz, D., y Constantinou, M. (2008). Development, Implementation and Verification of Dynamic Analysis Models for Multi-Spherical Sliding Bearings. Report No. MCEER-08-0018, University at Buffalo, State University of New York, Department of Civil, Structural and Environmental Engineering , Buffalo.

7. Fenz, D., y Constantinou, M. (2008). Mechanical Behavior of Multi-Spherical Sliding Bearings. Technical Report MCEER-08-0007, University at Buffalo, The State University of New York, Multidisciplinary Center for Earhquake Engineering Research.

8. Freire, y Ortíz. (2014). Análisis no lineal de estructuras. Tesis de grado para obtener el título de Ing. Civil, Universidad de las Fuerzas Armadas ESPE.

9. Guaygua , B. (2015). Análisis experimental y analítico de aisladores elastoméricos. Tesis para obtener el grado de Magister en Estructuras y Ciencias de los Materiales, Universidad Central del Ecuador, Quito. 
10. Gutiérrez , A. (2016). Deducción del Factor de Amortiguamiento en el modelo real de la Fase II. Universidad de las Fuerzas Armadas-ESPE, Curso de Análisis Sísmico .

11. McVitty, W., y Constantinou, M. (2015). Property Modification Factors for Seismic Isolators: Design Guidance for Buildings. Technical Report MCEER-15-0005, University at Buffalo, State University of New York.

12. Monge Romo, J. P. (2014). Análisis sísmico de una estructura con aisladores FPS de la primera y segunda generación. Tesis de grado para obtener el título de Ing. Civil, Universidad de las Fuerzas Armadas ESPE.

13. Rivas, A., Aguiar, R., Benito, M. B., Gaspar, J., y Parra, H. (2014). Determinación el período de recurrencia y magnitud máxima para el control de las estructuras en el rango elástico ante un sismo asociado a las fallas inversas de Quito. Revista Internacional de Ingeniería de Estructuras, 19(2), 201-217.

14. Suárez , V. (2013). I Congreso Internacional Ciencias de la Tierra y la Construcción-Aisladores Sísmicos. Edificio de la sede de la Unión de Naciones Sudamericanas (UNASUR). Sangolquí.

15. Vergara Navarrete, C. (2015). Estudio Comparativo de Aisladores Sísmicos FPS de Primera y Segunda Generación. Tesis para obtener el grado de Magister en Estructuras y Ciencias de los Materiales, Universidad Central del Ecuador, Quito. 